\title{
Spatial Diversification of Situation of the Organic Farming in the Polish Voivodeships in the Years 2010-2018
}

\author{
Konrad Podawca ${ }^{1 *}$, Norbert Dąbkowski ${ }^{2}$ \\ 1 Institute of Environmental Engineering, Faculty of Civil and Environmental Engineering, Warsaw University \\ of Life Sciences - SGGW, Nowoursynowska Street 159, 02-776 Warsaw, Poland \\ 2 Institute of Civil Engineering, Faculty of Civil and Environmental Engineering, Warsaw University of Life \\ Sciences - SGGW, Nowoursynowska Street 159, 02-776 Warsaw, Poland \\ *CCorresponding author's e-mail: konrad_podawca@sggw.pl
}

\begin{abstract}
The paper raises the problem of changes in situation of the organic farming in Poland. A set of features and indexes characterizing development or recession of the organic farms in individual voivodeships has been worked out. The authors used data for the years 2010-2018, made available in the Local Data Bank, and reports on the state of the organic farms in Poland. Quantitative and areal changes have been presented, concerning firstly the organic farms in relation to all farms as well as the agricultural area and, secondly, certified farms in relation to the organic farms. Using the arithmetic mean of synthetic indexes, the evaluation results have been compared to the synthetic index of usefulness for organic production which was worked out in the Institute of Soil Science and Plant Cultivation (IUNG) in Pulawy. A diversification of the voivodeships has been presented in terms of recession or development of the organic farms. Regions have been also selected where the negative trend of the phenomenon is opposite to that indicated by advantageous conditions for the organic farming. Examples of such voivodeships are Silesian, Holy Cross or Lower Silesian. The performed analysis proves that an interest in the organic farming in Poland significantly decreased in recent years. One can find the recession of the organic farming in 10 voivodeships, even at the level of 30-65\% in the Silesian, Holy Cross, Lesser Poland and Subcarpathia. The satisfactory development level of the organic farms through the recent 9 years has been stated only in three voivodeships: Lodz Province, Podlaskie, Warmian-Masurian.
\end{abstract}

Keywords: organic farming, development, recession, organic farm, voivodeship

\section{INTRODUCTION}

The origins of the organic farming date back to 1920 s. In that time the Polish workers knew about the alternative methods of farming owing to the count and countess von Keyserling who introduced in their farm a biodynamic method developed by Dr Steiner [Duda-Krynicka and Jaskólecki 2010]. However, 1984 is recognized as the beginning of the eco-agricultural movement in Poland. From the very onset, it was such form of farming which had to benefit not only farmers but, first of all, consumers and the environment. Thus, the development of farms promoting ecological cultivation and breeding became the subject of research [Zegar 2009].
In the source literature, the items presenting diversification in the development pace of the organic farms in various regions of the world, Europe and Poland can be found [Runowski 2009, Ligenzowska 2014, Drabarczyk and WrzesińskaKowal 2015, Golik and Żmija 2017]. Of course, it is affected, i.a. by the environmental conditions, natural quality of the agricultural space as well as financial aspects of the organic farming and market of ecological products [Kowalska 2010]. The majority of authors limit themselves to the quantitative characteristics [Komorowska 2007, Drabarczyk and Wrzesińska-Kowal 2015, Golik and Żmija 2017, Raport...] and tries to analyze it with the use of graphical methods (e.g. Lorentz's concentration curve) or analytical ones (Gini's 
coefficient) [Makowska et al. 2015]. It must be emphasized as well that many works concern the situation in Poland [Komorowska 2007]. The spatial characteristics are presented mostly in relation to the voivodeships, rarely in relation to smaller units, as counties [Kacprzak and Kołodziejczak 2011, Dąbkowski and Podawca 2017].

Till 2013, one can distinguish three periods in the development of the organic farming in Poland. Till 1999, when a financial support of that farming system did not exist, there were few of such farms - only 555, most of which were localized in the Holy Cross, Lublin and Mazovia voivodeships, while the least in the Kuyavia-Pomerania, Subcarpathia and Opole [Komorowska 2007]. When the payments for ecological areas were introduced in 2001 and the government started to refund the certification costs from the state budget in 2000-2004, the popularity of that form of farming increased [Łuczka-Bakuła 2013]. In 2004, an almost 8-fold increase of the organic farms related to 1999 (from 555 to 3760) was observed in Poland. In this period, the most organic farms were registered in the Lesser Poland, Holy Cross and Mazovia voivodeships. In 2013, however, the total number of the ecological farms in Poland amounted 26 598, what means a 7-fold increase in the number observed in 2004. Most farms were placed in the Warmia-Masuria, Podlasie and Mazovia voivodeships [Makowska 2015].

\section{OBJECTIVES OF THE ANALYSIS}

The main objective of the analysis was a presentation of the diversification of parameters characterizing the organic farming in individual voivodeships. The state of the organic farming has been described by a set of indices which constitutes a technique for assessing the development level of this method of agriculture in the voivodeships. It was assumed that the timespan of the analysis are the years 2010-2018.

The complementary objectives were:

- presentation of diversification of amount of the organic farms in each voivodeship,

- presentation of diversification of the voivodeships in terms of the area of organic agriculture.

Apart of the quantitative comparison, a time comparison has been worked out. The presented material allows observing the change trends occurring year by year in the individual voivodeships in relation to each parameter characterizing the organic farming.

\section{METHODS}

The following data were assumed to characterize the organic farms in Poland:

- amount of the organic farms $\mathrm{L}_{\text {of }}$ [items],

- amount of the certified organic farms $\mathrm{L}_{\text {cof }}$ [items],

- total amount of farms $\mathrm{L}_{\mathrm{af}}$ [items],

- area of the organic farms $\mathrm{A}_{\text {of }}$ [ha],

- area of the certified organic farms $\mathrm{A}_{\text {cof }}$ [ha],

- total area of farms $\mathrm{A}_{\mathrm{af}}$ [ha].

The following data were assumed with order to evaluate the diversification in the organic farming in terms of the area of farms as well as to compare the administrative units in these terms:

- share of the organic farms in the set of all farms $\mathrm{C}_{\text {of }}[-]$,

- share of the certified organic farms in the set of the organic farms $\mathrm{C}_{\text {cof }}[-]$,

- share of the area of the organic farms in the total agricultural area $\mathrm{C}_{\text {aof }}[-]$,

- share of the area of the certified organic farms in the area of the organic farms $\mathrm{C}_{\text {acof }}$ [ha].

The changes of the following data within the 9-year period 2010-2018, in the annual time modules, were used to present the development dynamics of the organic farming:

- increase or decrease of the area of the organic farms $\Delta \mathrm{A}_{\text {aof }}[\mathrm{ha}]$,

- increase or decrease of the amount of the organic farms $\Delta \mathrm{L}_{\text {of }}$ [items],

- increase or decrease of the area of the certified organic farms $\Delta \mathrm{A}_{\text {acof }}[$ ha],

- increase or decrease of the amount of the certified organic farms $\Delta \mathrm{L}_{\text {cof }}$ [items].

The following indices, related both to the amount and the area of the organic farms, were used to evaluate a development level in the organic farming within the period 2010-2018:

$$
\begin{aligned}
& \mathrm{W}_{\text {devof }}=\left(\mathrm{L}_{\text {ofmax }}-\mathrm{L}_{\text {of } 2010}\right) / \mathrm{L}_{\text {ofmax }}[-] ; \\
& \mathrm{W}_{\text {recof }}=\left(\mathrm{L}_{\text {ofmax }}-\mathrm{L}_{\text {of } 2018}\right) / \mathrm{L}_{\text {ofmax }}[-] ; \\
& \mathrm{W}_{\text {devaof }}=\left(\mathrm{A}_{\text {ofmax }}-\mathrm{A}_{\text {of } 2010}\right) / \mathrm{A}_{\text {offmax }}[-] ; \\
& \mathrm{W}_{\text {recaof }}=\left(\mathrm{A}_{\text {ofmax }}-\mathrm{A}_{\text {of2018 }}\right) / \mathrm{A}_{\text {ofmax }}[-] .
\end{aligned}
$$


It was caused by a fact that in each case one can determine a maximum of the amount of the organic farms and, usually, two periods - development and recession of the organic farms. In order to depict synthetically the diversification of the voivodeships in terms of the situation in the organic farming, synthetic indices were used, which constitute a difference between the periods of development and recession:

$$
\begin{aligned}
& \mathrm{W}_{\text {synt1 }}=\mathrm{W}_{\text {devof }}-\mathrm{W}_{\text {recof }}[-] ; \\
& \mathrm{W}_{\text {synt2 }}=\mathrm{W}_{\text {devaof }}-\mathrm{W}_{\text {recaof }}[-] .
\end{aligned}
$$

The analysis and interpretation of the investigation results were followed according to the stages below:

- filtration of the data gathered in the Local Data Bank (BDL) with use of the features contained in the category Agriculture, forestry and hunting, the subgroup Organic farms and Farms, in terms of areal groups of agricultural lands;

- data aggregation for individual subsets;

- dynamic data analysis (in one-year periods) with presentation of a trend of the changes.

Compilation of the numerical data in spatial terms has been worked out with use of the data from the BDL and the ArcGis software.

\section{CHARACTERISTICS OF DEVELOPMENT OF ORGANIC FARMS}

The data and features concerning the organic and certified farms are gathered in Table 1.

Table 1. Data and features concerning certified farms along with the change trend for the voivodeships in the

\begin{tabular}{|c|c|c|c|c|c|c|c|c|c|c|c|c|c|c|c|}
\hline Voi. & Year & $L_{f}$ & $A_{a f}$ & $\mathrm{~L}_{\text {of }}$ & $\Delta \mathrm{L}_{\text {of }}$ & $A_{\text {of }}$ & $\Delta \mathrm{A}_{\text {of }}$ & $\mathrm{L}_{\text {cof }}$ & $\Delta \mathrm{L}_{\text {cof }}$ & $A_{\text {acof }}$ & $\Delta \mathrm{A}_{\text {acof }}$ & $\mathrm{C}_{\text {of }}$ & $\mathrm{C}_{\text {cof }}$ & $\mathrm{C}_{\text {aof }}$ & $\mathrm{C}_{\text {acof }}$ \\
\hline \multirow{9}{*}{ 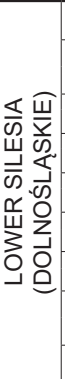 } & 2010 & 61834 & 995916 & 1227 & 95 & 39703 & 5844 & 778 & 136 & 5476 & 4555 & 0.0198 & 0.634 & 0.0399 & 0.6417 \\
\hline & 2011 & --- & 1072649 & 1322 & \multirow{2}{*}{$\begin{array}{c}-10 \\
-123\end{array}$} & 45547 & \multirow{2}{*}{-1243} & 914 & 127 & 30031 & \multirow{2}{*}{4392} & --- & 0.691 & 0.0425 & 0.6593 \\
\hline & 2012 & 61099 & 1084896 & 1312 & & 44304 & & 1041 & -32 & 34423 & & 0.0215 & 0.793 & 0.0408 & 0.7770 \\
\hline & 2013 & 59544 & 976338 & 1189 & \multirow{2}{*}{-143} & 37455 & -8040 & 1009 & -52 & 30972 & -3451 & 0.0200 & 0.849 & 0.0384 & 0.8269 \\
\hline & 2014 & --- & 994492 & 1046 & & 37005 & -450 & 954 & -53 & 33594 & $20 \angle 2$ & --- & 0.912 & 0.0372 & 0.9078 \\
\hline & 2015 & --- & 958588 & 849 & -191 & 31261 & -5 & 773 & 81 & 27622 & -5912 & --- & 0.910 & 0.0326 & 0.8836 \\
\hline & 2016 & 55993 & 950243 & 813 & -36 & 29200 & 1650 & 657 & 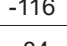 & 23854 & -3768 & 0.0145 & 0.808 & 0.0307 & 0.8169 \\
\hline & 2017 & --- & 964719 & 741 & $-1 / 2$ & 27542 & 058 & 573 & -84 & 21605 & $\angle 49$ & --- & 0.773 & 0.0285 & 0.7844 \\
\hline & 2018 & --- & 898865 & 713 & -28 & 27357 & -185 & 578 & 5 & 21002 & -603 & --- & 0.811 & 0.0304 & 0.7677 \\
\hline & 2010 & 68148 & 1233609 & 327 & 44 & 7688 & 688 & 232 & 27 & 6150 & 345 & 0.0048 & 0.709 & 0.0062 & 0.7999 \\
\hline$\S \frac{\omega}{\bar{c}}$ & 2011 & --- & 1227665 & 371 & 19 & 8376 & 437 & 259 & 38 & 6495 & 461 & --- & 0.698 & 0.0068 & 0.7754 \\
\hline 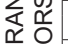 & 2012 & 64770 & 1132881 & 390 & 25 & 8813 & $\frac{431}{2339}$ & 297 & 30 & 6956 & 1969 & 0.0060 & 0.762 & 0.0078 & 0.7893 \\
\hline$\sum_{\Sigma}^{\mathrm{U}} \sum_{0}$ & 2013 & 65115 & 1140071 & 415 & -14 & 11152 & 421 & 341 & 20 & 8925 & 1321 & 0.0064 & 0.822 & 0.0098 & 0.8003 \\
\hline $\begin{array}{ll}2 & 0 \\
0 & 0 \\
0 & 0\end{array}$ & 2014 & --- & 1149709 & 401 & -38 & 11573 & $\begin{array}{l}4 \angle 1 \\
-928\end{array}$ & 361 & $\frac{20}{-31}$ & 10246 & $\begin{array}{l}13<1 \\
-751\end{array}$ & --- & 0.900 & 0.0101 & 0.8853 \\
\hline 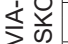 & 2015 & --- & 1147637 & 363 & $-3-2 y$ & 10645 & $-9<0$ & 330 & -38 & 9495 & -121 & --- & 0.909 & 0.0093 & 0.8920 \\
\hline 文 & 2016 & 63830 & 1107332 & 470 & & 9263 & & 292 & -32 & 7753 & 5 & 0.0074 & 0.621 & 0.0084 & 0.8370 \\
\hline २े & 2017 & --- & 1147008 & 419 & & 8331 & & 260 & & 6198 & & --- & 0.621 & 0.0073 & 0.7440 \\
\hline & 2018 & --- & 1183952 & 395 & -24 & 7655 & -676 & 285 & 25 & 5781 & -417 & --- & 0.722 & 0.0065 & 0.7552 \\
\hline & 2010 & 188266 & 1599705 & 1962 & 103 & 34855 & -18 & 1386 & 83 & 23539 & 1178 & 0.0104 & 0.706 & 0.0218 & 0.6753 \\
\hline & 2011 & --- & 1650534 & 2065 & 109 & 34837 & 2629 & 1469 & 144 & 24717 & 3447 & -- & 0.711 & 0.0211 & 0.7095 \\
\hline 岂 & 2012 & 177525 & 1614948 & 2174 & 109 & 37466 & $\angle 0 \angle 9$ & 1613 & $\begin{array}{l}144 \\
98\end{array}$ & 28164 & 3447 & 0.0122 & 0.742 & 0.0232 & 0.7517 \\
\hline उo & 2013 & 178135 & 1584383 & 2129 & & 40819 & & 1711 & 65 & 31351 & & 0.0120 & 0.804 & 0.0258 & 0.7680 \\
\hline 뭄 & 2014 & --- & 1584924 & 1975 & & 38467 & & 1776 & - & 32622 & & -- & 0.899 & 0.0243 & 0.8481 \\
\hline$\underline{z} \stackrel{m}{د}$ & 2015 & --- & 1643830 & 1825 & & 34052 & -2 & 1643 & 6 & 29586 & & --- & 0.900 & 0.0207 & 0.8688 \\
\hline 号己 & 2016 & 179994 & 1628626 & 1980 & -76 & 31343 & $\begin{array}{l}-2109 \\
-2342\end{array}$ & 1487 & -100 & 25187 & -2249 & 0.0110 & 0.751 & 0.0192 & 0.8036 \\
\hline & 2017 & --- & 1647054 & 1904 & & 29001 & & 1387 & & 22938 & & --- & 0.728 & 0.0176 & 0.7909 \\
\hline & 2018 & --- & 1614774 & 1948 & 44 & 28428 & -573 & 1466 & 79 & 22682 & -256 & --- & 0.753 & 0.0176 & 0.7979 \\
\hline & 2010 & 22147 & 490418 & 833 & 248 & 35797 & 8462 & 417 & 143 & 19297 & 4570 & 0.0376 & 0.501 & 0.0730 & 0.5391 \\
\hline & 2011 & --- & 521539 & 1081 & & 44259 & 832 & 560 & & 23867 & & -- & 0.518 & 0.0849 & 0.5393 \\
\hline & 2012 & 22354 & 515376 & 1356 & 66 & 52581 & 032 & 772 & 151 & 31610 & 11 & 0.0607 & 0.569 & 0.1020 & 0.6012 \\
\hline >ow & 2013 & 21256 & 456736 & 1422 & -52 & 54692 & -1392 & 923 & 210 & 35500 & 8914 & 0.0669 & 0.649 & 0.1197 & 0.6491 \\
\hline 茄 & 2014 & -- & 468979 & 1370 & -168 & 53300 & -6957 & 1133 & -77 & 44414 & -5075 & --- & 0.827 & 0.1137 & 0.8333 \\
\hline 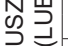 & 2015 & --- & 419363 & 1202 & -5 & 46343 & -3108 & 1056 & -136 & 39339 & -6696 & --- & 0.879 & 0.1105 & 0.8489 \\
\hline$\underline{\underline{m}}$ & 2016 & 20236 & 423777 & 1148 & & 43235 & -5312 & 920 & $\begin{array}{l}-100 \\
-214\end{array}$ & 32643 & -5320 & 0.0567 & 0.801 & 0.1020 & 0.7550 \\
\hline & 2017 & --- & 414919 & 948 & & 37923 & 740 & 706 & & 27323 & -0 & --- & 0.745 & 0.0914 & 0.7205 \\
\hline & 2018 & --- & 408942 & 877 & & 37174 & & 646 & -6 & 26651 & -672 & --- & 0.737 & 0.0909 & 0.7169 \\
\hline
\end{tabular}
years 2010-2018 (by authors) 
Table 1. cont.

\begin{tabular}{|c|c|c|c|c|c|c|c|c|c|c|c|c|c|c|c|}
\hline Voi. & Year & $L_{f}$ & $A_{a f}$ & $\mathrm{~L}_{\text {of }}$ & $\Delta \mathrm{L}_{\text {of }}$ & $A_{\text {of }}$ & $\Delta \mathrm{A}_{\text {of }}$ & $\mathrm{L}_{\text {cof }}$ & $\Delta \mathrm{L}_{\text {cof }}$ & $A_{\text {acof }}$ & $\Delta \mathrm{A}_{\text {acof }}$ & $\mathrm{C}_{\text {of }}$ & $\mathrm{C}_{\text {cof }}$ & $\mathrm{C}_{\text {aof }}$ & $\mathrm{C}_{\text {acof }}$ \\
\hline \multirow{9}{*}{ 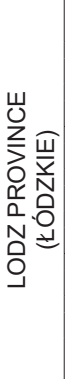 } & 2010 & 130565 & 1120552 & 420 & 58 & 7671 & 1075 & 234 & 64 & 3681 & 1036 & 0.0032 & 0.557 & 0.0068 & 0.4799 \\
\hline & 2011 & --- & 1115300 & 478 & \multirow{2}{*}{4} & 8746 & \multirow{2}{*}{1162} & 298 & \multirow{2}{*}{42} & 4717 & \multirow{2}{*}{881} & --- & 0.623 & 0.0078 & 0.5393 \\
\hline & 2012 & 130241 & 1113600 & 518 & & 9908 & & 340 & & 5598 & & 0.0040 & 0.656 & 0.0089 & 0.5650 \\
\hline & 2013 & 128309 & 1112531 & 528 & 10 & 10342 & $\begin{array}{l}434 \\
887\end{array}$ & 396 & 56 & 7238 & 1640 & 0.0041 & 0.750 & 0.0093 & 0.6999 \\
\hline & 2014 & --- & 1100298 & 508 & & 11229 & Sor & 423 & & 8753 & & -- & 0.833 & 0.0102 & 0.7795 \\
\hline & 2015 & --- & 1103135 & 478 & 19 & 10158 & -172 & 410 & -37 & 8437 & -646 & --- & 0.858 & 0.0092 & 0.8306 \\
\hline & 2016 & 124032 & 1096017 & 497 & -20 & 9986 & 112 & 373 & & 7791 & (0) & 0.0040 & 0.751 & 0.0091 & 0.7802 \\
\hline & 2017 & --- & 1160405 & 477 & & 9260 & & 354 & & 7431 & & --- & 0.742 & 0.0080 & 0.8025 \\
\hline & 2018 & --- & 1134572 & 491 & 14 & 8905 & -355 & 371 & 17 & 6955 & -476 & --- & 0.756 & 0.0078 & 0.7810 \\
\hline & 2010 & 153771 & 744222 & 2156 & -18 & 21968 & -572 & 1729 & 179 & 17835 & 839 & 0.0140 & 0.802 & 0.0295 & 0.8119 \\
\hline & 2011 & --- & 811118 & 2138 & -35 & 21396 & -346 & 1908 & 3 & 18674 & & -- & 0.892 & 0.0264 & 0.8728 \\
\hline 号邑 & 2012 & 152176 & 710093 & 2103 & V & 21050 & 640 & 1911 & 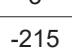 & 18890 & 210 & 0.0138 & 0.909 & 0.0296 & 0.8974 \\
\hline ङ & 2013 & 142874 & 685394 & 1838 & -460 & 17005 & -1476 & 1696 & -389 & 15208 & -800 & 0.0129 & 0.923 & 0.0248 & 0.8943 \\
\hline $\begin{array}{ll}0 & \overline{0} \\
\alpha & 0\end{array}$ & 2014 & --- & 649894 & 1378 & -700 & 15529 & טודו- & 1307 & -800 & 14408 & 000 & --- & 0.948 & 0.0239 & 0.9278 \\
\hline W & 2015 & --- & 663461 & 1128 & -35 & 12976 & -612 & 1070 & -125 & 11689 & -1050 & -- & 0.949 & 0.0196 & 0.9008 \\
\hline 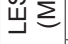 & 2016 & 139923 & 678802 & 1093 & 00 & 12364 & ST & 945 & 120 & 10639 & 1000 & 0.0078 & 0.865 & 0.0182 & 0.8605 \\
\hline & 2017 & --- & 687629 & 934 & & 10691 & & 805 & & 9275 & & --- & 0.862 & 0.0155 & 0.8676 \\
\hline & 2018 & --- & 669068 & 770 & -164 & 8844 & -1847 & 674 & -131 & 7548 & -1727 & --- & 0.875 & 0.0132 & 0.8535 \\
\hline & 2010 & 228821 & 2266491 & 1935 & 205 & 46229 & 3871 & 1232 & 177 & 25026 & 6076 & 0.0085 & 0.637 & 0.0204 & 0.5413 \\
\hline & 2011 & --- & 1328186 & 2140 & 233 & 50100 & 5704 & 1409 & 246 & 31102 & 8110 & -- & 0.658 & 0.0377 & 0.6208 \\
\hline Щ्ञ & 2012 & 234503 & 2339276 & 2373 & 24 & 55804 & 7641 & 1655 & 291 & 39212 & 8170 & 0.0101 & 0.697 & 0.0239 & 0.7027 \\
\hline$\$ \frac{y}{0}$ & 2013 & 212159 & 2207883 & 2609 & -235 & 63445 & -3091 & 1946 & 78 & 45989 & 4497 & 0.0123 & 0.746 & 0.0287 & 0.7249 \\
\hline 岕 & 2014 & --- & 2160781 & 2374 & & 60354 & ( & 2024 & 10 & 50486 & וסדד & -- & 0.853 & 0.0279 & 0.8365 \\
\hline$\sum$ 这 & 2015 & --- & 2248532 & 2147 & $\begin{array}{l}-221 \\
279\end{array}$ & 53790 & $\begin{array}{l}-6504 \\
-4273\end{array}$ & 1860 & -169 & 44827 & -6093 & -- & 0.866 & 0.0239 & 0.8334 \\
\hline & 2016 & 212917 & 2211447 & 2426 & 210 & 49517 & The & 1691 & -184 & 38734 & 0000 & 0.0114 & 0.697 & 0.0224 & 0.7822 \\
\hline & 2017 & --- & 2244490 & 2215 & & 44348 & & 1507 & & 33883 & & --- & 0.680 & 0.0198 & 0.7640 \\
\hline & 2018 & --- & 2474522 & 2284 & 69 & 42049 & -2299 & 1593 & 86 & 32064 & -1819 & --- & 0.697 & 0.0170 & 0.7625 \\
\hline & 2010 & 28437 & 538712 & 79 & 7 & 3180 & -477 & 49 & 6 & 2190 & -603 & 0.0028 & 0.620 & 0.0059 & 0.6887 \\
\hline & 2011 & --- & 555283 & 86 & 4 & 2703 & 2 & 55 & 11 & 1587 & 229 & --- & 0.640 & 0.0049 & 0.5871 \\
\hline U & 2012 & 26832 & 543306 & 90 & $\begin{array}{l}4 \\
-2\end{array}$ & 2930 & $\frac{221}{613}$ & 66 & 8 & 1816 & 762 & 0.0034 & 0.733 & 0.0054 & 0.6198 \\
\hline ठ․ & 2013 & 26753 & 571488 & 88 & -13 & 3543 & -237 & 74 & -5 & 2578 & -97 & 0.0033 & 0.841 & 0.0062 & 0.7276 \\
\hline$\frac{\alpha}{2} \frac{0}{0}$ & 2014 & --- & 510019 & 75 & -8 & 3306 & -264 & 69 & -11 & 2481 & -247 & -- & 0.920 & 0.0065 & 0.7505 \\
\hline Ш & 2015 & --- & 520303 & 67 & 1 & 3042 & 174 & 58 & -1 & 2234 & 119 & -- & 0.866 & 0.0058 & 0.7344 \\
\hline & 2016 & 26919 & 533060 & 68 & -11 & 3216 & & 57 & -9 & 2353 & -1 & 0.0025 & 0.838 & 0.0060 & 0.7317 \\
\hline & 2017 & --- & 529825 & 57 & 11 & 2790 & & 48 & $-y$ & 2189 & -104 & - & 0.842 & 0.0053 & 0.7846 \\
\hline & 2018 & --- & 524200 & 61 & 4 & 3554 & 764 & 50 & 2 & 2501 & 312 & --- & 0.820 & 0.0068 & 0.7037 \\
\hline & 2010 & 140465 & 723600 & 2091 & -46 & 31868 & 491 & 1620 & 104 & 24868 & 1467 & 0.0149 & 0.775 & 0.0440 & 0.7803 \\
\hline & 2011 & --- & 772538 & 2045 & -1 & 32359 & -191 & 1724 & -18 & 26335 & -26 & -- & 0.843 & 0.0419 & 0.8138 \\
\hline$\lesssim \widetilde{\Psi}$ & 2012 & 134024 & 748495 & 1940 & -190 & 30381 & -875 & 1706 & -133 & 26309 & -429 & 0.0145 & 0.879 & 0.0406 & 0.8660 \\
\hline 志志 & 2013 & 132823 & 686671 & 1750 & -275 & 29506 & -5996 & 1573 & -208 & 25880 & -5213 & 0.0132 & 0.899 & 0.0430 & 0.8771 \\
\hline 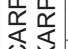 & 2014 & --- & 678969 & 1475 & -214 & 23510 & -6854 & 1365 & -234 & 20667 & -6176 & 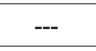 & 0.925 & 0.0346 & 0.8791 \\
\hline 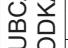 & 2015 & --- & 670218 & 1261 & -9 & 16656 & -1170 & 1131 & -112 & 14491 & -1585 & --- & 0.897 & 0.0249 & 0.8700 \\
\hline कळ & 2016 & 132851 & 686614 & 1252 & -58 & 15486 & -137 & 1019 & -107 & 12906 & -381 & 0.0094 & 0.814 & 0.0226 & 0.8334 \\
\hline & 2017 & -- & 683730 & 1194 & & 15349 & & 912 & & 12525 & & --- & 0.764 & 0.0224 & 0.8160 \\
\hline & 2018 & --- & 645746 & 1131 & -63 & 13630 & -1719 & 927 & 15 & 11435 & -1090 & --- & 0.820 & 0.0211 & 0.8390 \\
\hline & 2010 & 84136 & 1260925 & 2033 & 407 & 42917 & 9149 & 1038 & 379 & 18890 & 11251 & 0.0242 & 0.511 & 0.0340 & 0.4402 \\
\hline & 2011 & --- & 1271067 & 2440 & 484 & 52066 & 4301 & 1417 & 494 & 30141 & 9032 & --- & 0.581 & 0.0410 & 0.5789 \\
\hline$\stackrel{2}{\gtrless}$ & 2012 & 80873 & 1286687 & 2924 & 483 & 56367 & 7181 & 1911 & 324 & 39173 & 5635 & 0.0362 & 0.654 & 0.0438 & 0.6950 \\
\hline 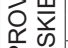 & 2013 & 79083 & 1256787 & 3407 & 25 & 63548 & 1349 & 2235 & 345 & 44808 & 649 & 0.0431 & 0.656 & 0.0506 & 0.7051 \\
\hline 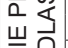 & 2014 & -- & 1264636 & 3432 & -159 & 64897 & -8369 & 2580 & 235 & 51300 & -2747 & --- & 0.752 & 0.0513 & 0.7905 \\
\hline क्ष & 2015 & --- & 1243342 & 3273 & 164 & 56528 & -1360 & 2815 & -87 & 48553 & -2480 & --- & 0.860 & 0.0455 & 0.8589 \\
\hline 光 & 2016 & 81181 & 1277656 & 3437 & -226 & 55168 & -1617 & 2728 & -152 & 46073 & -1058 & 0.0423 & 0.794 & 0.0432 & 0.8351 \\
\hline & 2017 & --- & 1263799 & 3211 & & 53551 & & 2576 & & 45015 & & -- & 0.802 & 0.0424 & 0.8406 \\
\hline & 2018 & --- & 1235832 & 2989 & & 51608 & -1 & 2420 & 6 & 41789 & 26 & --- & 0.810 & 0.0418 & 0.8097 \\
\hline
\end{tabular}


Table 1. cont.

\begin{tabular}{|c|c|c|c|c|c|c|c|c|c|c|c|c|c|c|c|}
\hline Voi. & Year & $L_{f}$ & $A_{a f}$ & $\mathrm{~L}_{\text {of }}$ & $\Delta \mathrm{L}_{\text {of }}$ & $A_{\text {of }}$ & $\Delta \mathrm{A}_{\text {of }}$ & $\mathrm{L}_{\text {cof }}$ & $\Delta \mathrm{L}_{\text {cof }}$ & $A_{\text {acof }}$ & $\Delta \mathrm{A}_{\text {acof }}$ & $\mathrm{C}_{\text {of }}$ & $\mathrm{C}_{\text {cof }}$ & $\mathrm{C}_{\text {aof }}$ & $\mathrm{C}_{\text {acof }}$ \\
\hline \multirow{9}{*}{ 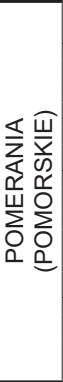 } & 2010 & 41136 & 859654 & 648 & 115 & 22554 & 4803 & 348 & 112 & 12048 & 4355 & 0.0158 & 0.537 & 0.0262 & 0.5342 \\
\hline & 2011 & --- & 895869 & 763 & \multirow{2}{*}{131} & \multirow{2}{*}{\begin{tabular}{|l|}
27357 \\
30615
\end{tabular}} & \multirow{2}{*}{3258} & 460 & 141 & 16403 & \multirow{2}{*}{4820} & -- & 0.603 & 0.0305 & 0.5996 \\
\hline & 2012 & 40035 & 907976 & 894 & & & & 601 & 141 & 21223 & & 0.0223 & 0.672 & 0.0337 & 0.6932 \\
\hline & 2013 & 39956 & 843240 & 893 & -46 & 28721 & 561 & 645 & 63 & 21146 & 4038 & 0.0223 & 0.722 & 0.0341 & 0.7363 \\
\hline & 2014 & --- & 822341 & 847 & (70 & 29282 & r & 708 & 政 & 25184 & (7000 & --- & 0.836 & 0.0356 & 0.8601 \\
\hline & 2015 & --- & 850604 & 737 & -58 & 24866 & -1538 & 642 & -74 & 21988 & -3222 & --- & 0.871 & 0.0292 & 0.8843 \\
\hline & 2016 & 39049 & 814774 & 679 & 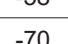 & 23328 & -1000 & 568 & & 18766 & \begin{tabular}{|l|}
-2268 \\
-2268
\end{tabular} & 0.0174 & 0.837 & 0.0286 & 0.8044 \\
\hline & 2017 & --- & 843223 & 609 & & 22419 & - & 497 & & 16498 & 2200 & --- & 0.816 & 0.0266 & 0.7359 \\
\hline & 2018 & --- & 833793 & 540 & -69 & 19974 & -2445 & 441 & -56 & 14675 & -1823 & --- & 0.817 & 0.0240 & 0.7347 \\
\hline & 2010 & 64745 & 447509 & 228 & 10 & 5739 & 1048 & 153 & 20 & 3451 & 801 & 0.0035 & 0.671 & 0.0128 & 0.6013 \\
\hline & 2011 & --- & 502643 & 238 & -2 & 6787 & 338 & 173 & 19 & 4252 & 1285 & --- & 0.727 & 0.0135 & 0.6265 \\
\hline & 2012 & 64803 & 436500 & 236 & 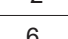 & 7125 & (2) & 192 & re & 5537 & 1200 & 0.0036 & 0.814 & 0.0163 & 0.7771 \\
\hline$\leq \overleftrightarrow{\bar{y}}$ & 2013 & 58981 & 434688 & 242 & $\begin{array}{c}0 \\
-12\end{array}$ & 7220 & 56 & 205 & 13 & 5661 & $\frac{124}{1257}$ & 0.0041 & 0.847 & 0.0166 & 0.7841 \\
\hline 岃宗 & 2014 & --- & 412525 & 230 & -29 & 7788 & -1150 & 207 & -34 & 6918 & -1096 & --- & 0.900 & 0.0189 & 0.8883 \\
\hline $\bar{\omega} \cdot \bar{\omega}$ & 2015 & --- & 400909 & 201 & -21 & 6638 & -1314 & 173 & -22 & 5822 & -1420 & --- & 0.861 & 0.0166 & 0.8771 \\
\hline & 2016 & 54503 & 415249 & 180 & -18 & 5324 & -1598 & 151 & -19 & 4402 & -1429 & 0.0033 & 0.839 & 0.0128 & 0.8268 \\
\hline & 2017 & --- & 4206006 & 162 & & 3726 & & 132 & & 2973 & & --- & 0.815 & 0.0009 & 0.7979 \\
\hline & 2018 & -- & 422867 & 148 & -14 & 2951 & -775 & 118 & -14 & 2301 & -672 & --- & 0.797 & 0.0070 & 0.7797 \\
\hline & 2010 & 96672 & 598764 & 1243 & 53 & 13123 & 1178 & 928 & 81 & 9270 & 1521 & 0.0129 & 0.747 & 0.0219 & 0.7064 \\
\hline & 2011 & --- & 604202 & 1296 & -8 & 14301 & 250 & 1009 & 68 & 10791 & 618 & --- & 0.779 & 0.0237 & 0.7546 \\
\hline$\frac{w}{\underline{x}}$ & 2012 & 92654 & 587411 & 1288 & - & 14551 & 200 & 1077 & 0 & 11409 & 13 & 0.0139 & 0.836 & 0.0248 & 0.7841 \\
\hline कृ & 2013 & 90241 & 575996 & 1207 & -215 & 15122 & -2084 & 1081 & $\begin{array}{c}4 \\
-148\end{array}$ & 12728 & -1218 & 0.0134 & 0.896 & 0.0263 & 0.8417 \\
\hline 岳 $\frac{r}{r}$ & 2014 & --- & 560124 & 992 & -210 & 13038 & ד & 933 & -140 & 11510 & -913 & -- & 0.941 & 0.0233 & 0.8828 \\
\hline 흐는 & 2015 & --- & 560702 & 853 & -19 & 11598 & -859 & 807 & -100 & 10597 & -1256 & --- & 0.946 & 0.0207 & 0.9137 \\
\hline I $\underset{\infty}{\infty}$ & 2016 & 85308 & 562031 & 834 & 4 & 10739 & -769 & 707 & -93 & 9341 & -998 & 0.0098 & 0.848 & 0.0191 & 0.8698 \\
\hline & 2017 & --- & 564757 & 740 & 4 & 9970 & -109 & 614 & -90 & 8343 & -950 & --- & 0.830 & 0.0177 & 0.8368 \\
\hline & 2018 & --- & 540019 & 680 & -60 & 9087 & -883 & 575 & -39 & 7618 & -725 & --- & 0.846 & 0.0168 & 0.8383 \\
\hline & 2010 & 43788 & 1144844 & 2279 & 754 & 75242 & 23231 & 989 & 449 & 34130 & 17450 & 0.0520 & 0.434 & 0.0657 & 0.4536 \\
\hline$\frac{\mathrm{w}}{\mathrm{v}}$ & 2011 & -- & 1186800 & 3033 & 760 & 98473 & 14472 & 1438 & 722 & 51580 & 16035 & --- & 0.474 & 0.0830 & 0.5238 \\
\hline$\stackrel{\mathscr{N}}{\stackrel{\Upsilon}{丂}}$ & 2012 & 43995 & 1158564 & 3793 & 442 & 112945 & 3254 & 2160 & 446 & 67615 & 6488 & 0.0862 & 0.569 & 0.0975 & 0.5987 \\
\hline$\stackrel{N}{\$}$ & 2013 & 41928 & 1143952 & 4235 & -1 & 116199 & 898 & 2606 & 590 & 74103 & $\begin{array}{c}0400 \\
16028\end{array}$ & 0.1010 & 0.615 & 0.1016 & 0.6377 \\
\hline 仓े & 2014 & --- & 1120247 & 4234 & -193 & 117097 & -4329 & 3196 & 281 & 90131 & 2818 & --- & 0.755 & 0.1045 & 0.7697 \\
\hline ڤ & 2015 & --- & 1096060 & 4041 & 101 & 112768 & -4101 & 3477 & -242 & 92949 & -7217 & --- & 0.860 & 0.1029 & 0.8242 \\
\hline$\sum_{\propto}$ & 2016 & 43165 & 1130329 & 4142 & & 108667 & ז & 3235 & & 85732 & & 0.0960 & 0.781 & 0.0961 & 0.7889 \\
\hline$\frac{1}{3}$ & 2017 & --- & 1053008 & 3745 & - & 107067 & -1000 & 2870 & & 80817 & & --- & 0.766 & 0.1017 & 0.7548 \\
\hline & 2018 & --- & 1075762 & 3393 & -352 & 104574 & -2493 & 2719 & -151 & 76076 & -4741 & --- & 0.801 & 0.0972 & 0.7275 \\
\hline & 2010 & 125692 & 1952281 & 748 & 140 & 32513 & 5921 & 456 & 78 & 20755 & 3640 & 0.0060 & 0.610 & 0.0167 & 0.6384 \\
\hline & 2011 & --- & 1968065 & 888 & 8 & 38434 & 3045 & 534 & 117 & 24395 & 4792 & -- & 0.601 & 0.0195 & 0.6347 \\
\hline 号岸 & 2012 & 123228 & 1974634 & 974 & 32 & 41479 & 138 & 651 & 111 & 29187 & 3180 & 0.0079 & 0.668 & 0.0210 & 0.7037 \\
\hline Oे & 2013 & 122788 & 1920099 & 1006 & -40 & 41617 & 454 & 762 & 97 & 32367 & 5111 & 0.0082 & 0.757 & 0.0217 & 0.7777 \\
\hline 品造 & 2014 & --- & 1937521 & 966 & -157 & 42071 & -7548 & 859 & -134 & 37478 & -6879 & --- & 0.889 & 0.0217 & 0.8908 \\
\hline 宸至 & 2015 & --- & 1881413 & 809 & 34 & 34523 & -5352 & 725 & -133 & 30599 & -7140 & --- & 0.896 & 0.0183 & 0.8863 \\
\hline 岩岕 & 2016 & 121157 & 1823856 & 843 & -107 & 29171 & -3782 & 592 & -109 & 23459 & -3796 & 0.0070 & 0.702 & 0.0160 & 0.8042 \\
\hline & 2017 & --- & 1925032 & 736 & -101 & 25389 & $-3 / 02$ & 483 & -109 & 19663 & -3190 & --- & 0.656 & 0.0132 & 0.7745 \\
\hline & 2018 & --- & 1878196 & 727 & -9 & 25994 & 605 & 511 & 28 & 18501 & -1162 & --- & 0.703 & 0.0138 & 0.7117 \\
\hline & 2010 & 30525 & 1008609 & 2373 & 692 & 98023 & 21757 & 1312 & 295 & 61491 & 9459 & 0.0777 & 0.553 & 0.0972 & 0.6273 \\
\hline$\varangle \frac{\dot{w}}{\omega}$ & 2011 & --- & 953611 & 3065 & 514 & 119780 & 15587 & 1607 & 587 & 70950 & 19016 & --- & 0.524 & 0.1256 & 0.5923 \\
\hline 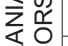 & 2012 & 28739 & 949169 & 3579 & 61 & 135367 & -5782 & 2194 & 475 & 89966 & 8552 & 0.1245 & 0.613 & 0.1426 & 0.6646 \\
\hline 妥边 & 2013 & 29062 & 891230 & 3640 & -11 & 129585 & -129 & 2669 & 456 & 98518 & 17189 & 0.1252 & 0.733 & 0.1454 & 0.7603 \\
\hline 으으. & 2014 & --- & 891858 & 3526 & -483 & 456 & -14569 & 3125 & -282 & 5707 & -12009 & - & 0.886 & 0.1452 & 0.8938 \\
\hline 战员。 & 2015 & -- & 889560 & 3043 & -470 & 114887 & -14317 & 2843 & -577 & 103698 & -22435 & -- & 0.934 & 0.1292 & 0.9026 \\
\hline щ온인. & 2016 & 29646 & 896383 & 2573 & -408 & 100570 & -12950 & 26 & -519 & 81263 & -14695 & 0.0868 & 0.881 & 0.1122 & 0.8080 \\
\hline N & 2017 & --- & 864623 & 2165 & & 87620 & & 1747 & & 66568 & -14000 & --- & 0.807 & 0.1013 & 0.7597 \\
\hline & 2018 & --- & 874354 & 2060 & -105 & 92892 & & 1553 & -194 & 65985 & -583 & --- & 0.754 & 0.1062 & 0.7103 \\
\hline
\end{tabular}


Looking at the data, one can observe that the highest amount of the organic farms corresponds to the years 2012-2013. This situation concerns the following voivodeships: Lublin, Lubusz, Mazovia, Opole, Pomerania, Silesia, Varmia-Masuria, Greater Poland and West Pomerania. The crisis in the development of the organic farms began earlier in the Lower Silesia, Lesser Poland, Subcarpathia and Holy Cross voivodeships. Only in the Kuyavia-Pomerania and Podlasie voivodeships this decline started later, i.e. in 2016. It can be observed that in most cases the decrease in the amount of the organic farms has been continuous for several years. Only in the Mazovia, Lodz and Lublin voivodeships it fluctuates i.e. in one year, their amount increases and in the next one it decreases.

It is also visible that the share of the organic farms in the set of all farms is not high. In five cases, i.e. Greater Poland, Silesia, Opole, Lodz and Kuyavia-Pomerania voivodeships, it does not even reach $1 \%$. In most voivodeships - in eight of them (Lower Silesia, Lublin, Lesser Poland, Mazovia, Subcarpathia, Holy Cross, Pomerania, West Pomerania) - it is at a level of $1 \div 2 \%$. Only in three cases, one can acknowledge that the form of organic farming constitutes a significant contribution to the agriculture: in the Varmia-Masuria, West Pomerania and Podlasie voivodeships.

The shares of the agricultural areas cultivated with ecological methods in relation to the overall agricultural area follow a similar pattern. These shares are only slightly higher than the shares concerning the amount of the farms. Thus, it can be concluded that an average organic farm is greater than an average regular farm in the given voivodeship.

A positive aspect of the organic farming development is the share of the certified farms and their areas in the set of all ecological farms. Regardless the voivodeship, this share falls within the range of $70-90 \%$. It proves an awareness of the organic farmers and need for the formalization of their activity and products.

\section{RESULTS}

It results clearly from the analysis that the recession in the situation in the organic farms is oncoming. In various voivodeships, this recession began in a different period, but mostly it was the year 2013. However, not only the very phenomenon of decrease in the number of the organic farms but also the intensity of this process in the years 2010-2018 must be taken into consideration (Fig. 1, Fig. 2). The indices presented in Table 2 show the dynamics of increase and decrease of the amount of the organic farms as well as the area covered by the organic farming.

The applied methodology allows showing clearly that, in most voivodeships, the recession indicators in the organic farming predominate the previous development indicators. The worst situation is observed in the Lesser Poland and Subcarpathia voivodeships where

Table 2. Indices of development and recession and synthetic indices of the organic farms in the voivodeships in the years 2010-2018

\begin{tabular}{|c|l|c|c|c|c|c|c|c|}
\hline No. & \multicolumn{1}{|c|}{ Voivoship } & $\mathrm{W}_{\text {devof }}$ & $\mathrm{W}_{\text {recof }}$ & $\mathrm{W}_{\text {devaof }}$ & $\mathrm{W}_{\text {recaof }}$ & $\mathrm{W}_{\text {synt1 }}$ & $\mathrm{W}_{\text {synt2 }}$ & $\mathrm{W}_{\text {synt3 }}$ \\
\hline 1 & Lower Silesia & 0.072 & 0.461 & 0.128 & 0.399 & -0.389 & -0.271 & -0.330 \\
\hline 2 & Kuyavia-Pomerania & 0.304 & 0.160 & 0.336 & 0.339 & 0.145 & -0.003 & 0.071 \\
\hline 3 & Lublin & 0.098 & 0.104 & 0.146 & 0.304 & -0.006 & -0.157 & -0.082 \\
\hline 4 & Lubusz & 0.414 & 0.383 & 0.345 & 0.320 & 0.031 & 0.025 & 0.028 \\
\hline 5 & Lodz & 0.205 & 0.070 & 0.317 & 0.207 & 0.134 & 0.110 & 0.122 \\
\hline 6 & Lesser Poland & 0.000 & 0.643 & 0.000 & 0.597 & -0.643 & -0.597 & -0.620 \\
\hline 7 & Masovia & 0.258 & 0.125 & 0.271 & 0.337 & 0.134 & -0.066 & 0.034 \\
\hline 8 & Opole & 0.122 & 0.322 & 0.102 & 0.003 & -0.200 & 0.100 & -0.050 \\
\hline 9 & Subcarpathia & 0.000 & 0.459 & 0.015 & 0.579 & -0.459 & -0.564 & -0.512 \\
\hline 10 & Podlasie & 0.408 & 0.130 & 0.339 & 0.205 & 0.278 & 0.134 & 0.206 \\
\hline 11 & Pomerania & 0.275 & 0.396 & 0.263 & 0.348 & -0.121 & -0.084 & -0.103 \\
\hline 12 & Silesia & 0.058 & 0.388 & 0.263 & 0.621 & -0.331 & -0.358 & -0.345 \\
\hline 13 & Holy Cross & 0.041 & 0.475 & 0.132 & 0.399 & -0.434 & -0.267 & -0.351 \\
\hline 14 & Warmia-Masuria & 0.462 & 0.199 & 0.357 & 0.107 & 0.263 & 0.250 & 0.257 \\
\hline 15 & Greater Poland & 0.256 & 0.277 & 0.227 & 0.382 & -0.021 & -0.155 & -0.088 \\
\hline 16 & West Pomerania & 0.348 & 0.434 & 0.276 & 0.314 & -0.086 & -0.038 & -0.062 \\
\hline
\end{tabular}




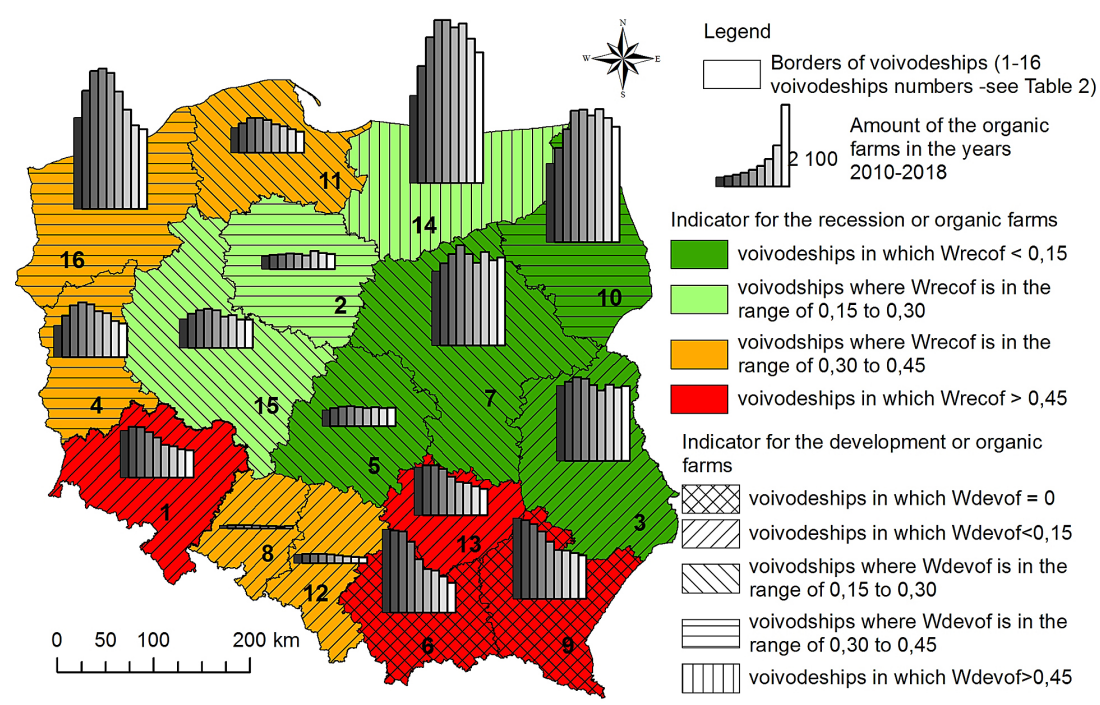

Fig. 1. Diversification of the voivodeships in terms of the changes in the amount of the organic farms in the years 2010-2018 (by authors)

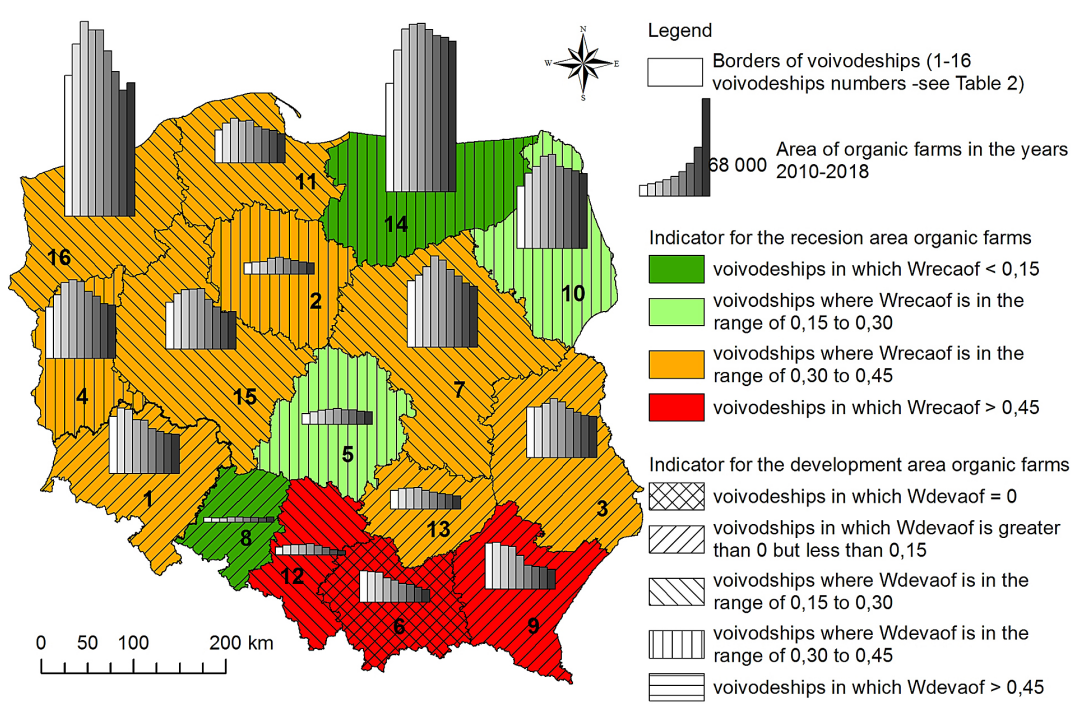

Fig. 2. Diversification of the voivodeships in terms of the changes in the area of the organic farms in the years 2010-2018 (by authors)

the amount of the organic farms has been increasing since 2010 . Only slightly better conditions exist in the Holy Cross, Silesia and Lower Silesia voivodeships (Fig. 1).

Despite the general decrease in the amount of the organic farms, in the case of the VarmiaMasuria, Podlasie and Lodz voivodeships the development indices are higher than the recession indices (Fig.1).

Two characteristic units were distinguished as well. In the Kuyavia-Pomerania and Masovia voivodeships the increase in the amount of the organic farms was higher than its decrease but, despite that, the agricultural area covered by the organic farms decreased. The reverse situation occurred in the Opole voivodeship where, despite the general decrease in the amount of the organic farms between 2010 and 2018 years, the area of the organic farms increased (Fig. 1, 2).

\section{DISCUSSION}

The abovementioned three development periods of the organic agriculture in Poland must be supplemented by a fourth period - the years 2013-2018. In these years, one can observe a recession in the popularity of this form of agriculture and decline of organic farming, both in terms of the amount and area. 
Table 3. Comparison of conditions for organic production with the recession in the organic farms in voivodships in years 2010-2018

\begin{tabular}{|c|c|c|c|c|c|c|}
\hline \multirow{2}{*}{\multicolumn{2}{|c|}{ Specification }} & \multicolumn{5}{|c|}{ Recession in the organic farming in years $2010-2018$} \\
\hline & & none & low & medium & high & very high \\
\hline \multirow{4}{*}{$\begin{array}{l}\text { Conditions for organic } \\
\text { production } \\
\text { [Stuczyński et al. } \\
2007]\end{array}$} & very favorable & Varmia-Masuria & - & - & - & - \\
\hline & favorable & $\begin{array}{c}\text { Kuyavia- } \\
\text { Pomerania }\end{array}$ & Lublin, Opole & Pomerania & $\begin{array}{c}\text { Lower Silesia, } \\
\text { Holy Cross }\end{array}$ & Subcarpathia \\
\hline & medium favorable & $\begin{array}{l}\text { Masovia, Lodz, } \\
\text { Podlasie, Lubusz }\end{array}$ & $\begin{array}{c}\text { West } \\
\text { Pomerania, } \\
\text { Greater Poland }\end{array}$ & - & 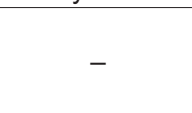 & - \\
\hline & unfavorable & - & - & - & Silesia & $\begin{array}{l}\text { Lesser } \\
\text { Poland }\end{array}$ \\
\hline
\end{tabular}

The obtained spatial diversification of the voivodeships with use of the arithmetic mean of synthetic indices $\left(\mathrm{W}_{\mathrm{synt}}\right)$ was compared to the synthetic index of usefulness for organic production which was worked out in the Institute of Soil Science and Plant Cultivation (IUNG-PIB) in Puławy [Biesiacki et al. 2004] (Fig. 3).

As one can note in the comparison, in extreme cases an analogy exists between the conditions for organic production and the recent development situation of this agriculture form. In the Warmia-Masuria voivodeship, having the best conditions, the amount and area of the organic farms within the investigated period is still growing. However, it must be emphasized that since 2014, a decrease trend has occurred, which is analogical to the general situation in Poland.
The greatest recession in establishing the organic farms occurred in the Lesser Poland and Silesia voivodeships. It is certainly connected to the disadvantageous conditions for the organic production in these regions. The situation in the voivodeships with medium advantage conditions for organic production can be considered as quite stable. In these regions, the development slightly predominates the recession (Mazovia, Lodz, Podlasie, Lubusz) or the recession is on a low level (West Pomerania, Greater Poland).

The most diversified group comprises the voivodeships with the advantageous conditions for organic production. There is no such relationship in this set as in the previously mentioned cases because one can find not only the voivodeships with no recession or low recession
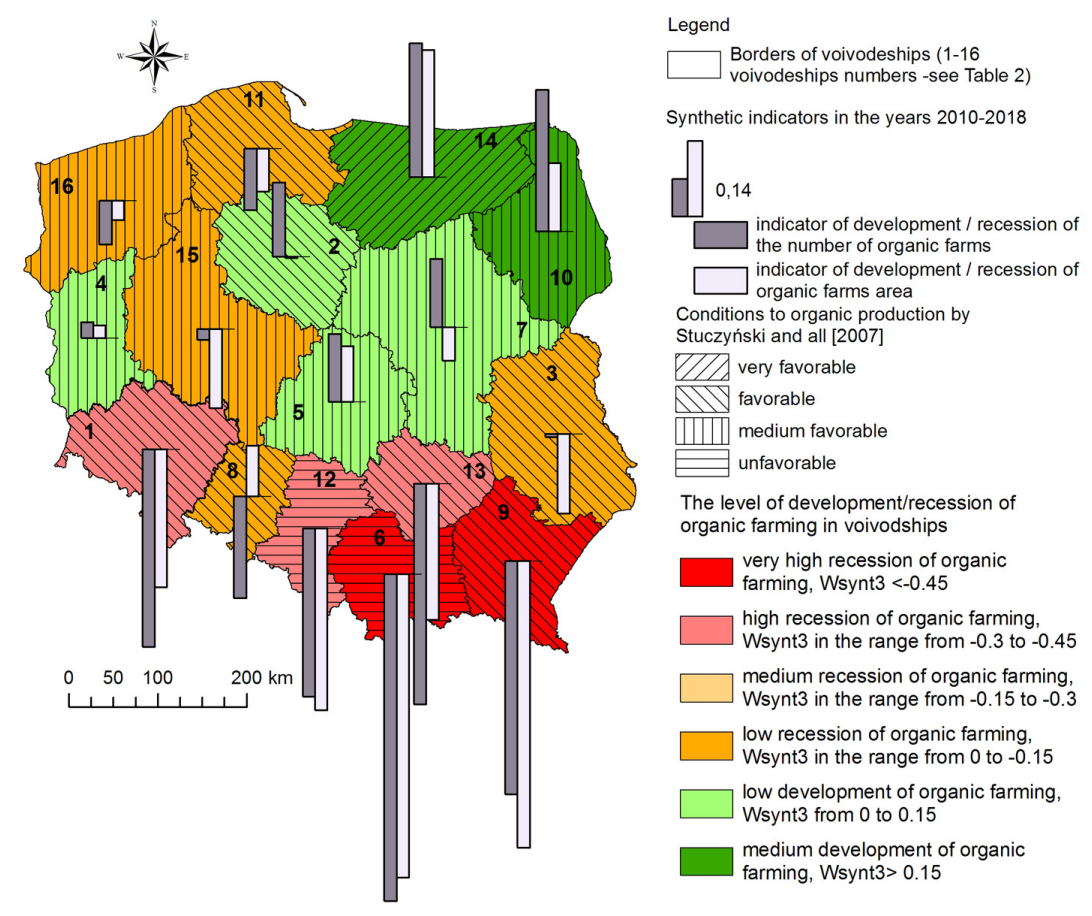

Fig. 3. Types of voivodeships in terms of usefulness for organic production and of recession / development of organic farms 
(Kuyavia-Pomerania, Lublin, Opole), but also with very significant recession, if compared to the previous periods (Lower Silesian, Holy Cross, Subcarpathia). Attention should be given to the worst situation in the southern voivodeships, mainly the mountain ones. The voivodeships: Kuyavia-Pomerania, Lublin, Opole, Pomerania, Lower Silesia, Holy Cross and Subcarpathia provide the evidence that the recession of the Polish agriculture can be of economic and social reasons, not only the environmental ones (Fig. 3).

\section{CONCLUSIONS}

The performed analysis proved that the statement being repeated in scientific works till 2015, according to which "the interest in the organic production in Poland is still growing" [Makowska 2015], has become false for the recent years.

The recession in the organic farming on various levels can be found in 10 voivodeships. The recession on a very high level of $30-65 \%$ occurs in the Silesian, Holy Cross, Lesser Poland and Subcarpathia voivodeships. The satisfactory development level of the organic farms within the 9-year period can be confirmed only for three voivodeships: Lodz, Podlasie and Warmia-Masuria.

The decrease of the interest in the organic form of agriculture, confirmed by the decreasing number of the existing organic farms, has been observed in all voivodeships - however, great differences in the intensity of this decrease can be noted, both in relation to the individual years and the voivodeships.

On the basis of the presented data, it can be stated that in the majority of the voivodeships, the crisis of the Polish organic farming began in the years 2012-2013. With regard to the spatial situation of the development of the organic farms in Poland, one can distinguish four characteristic regions:

- southern - with high or very high recession, where very dynamic fall in the organic farms occurred,

- northwestern - with medium recession of the organic farming,

- northeastern - where, despite the general fall of the amount of the organic farms, the development occurred within the years 2010-2018,

- central - where, despite the general fall of the amount of the organic farms as well, one can find a stable situation within the years 2010-2018.
In statistical and spatial terms, however, it must be stated that the percentage distribution of the existing organic farms in the voivodeships is quite stable within the years 2014-2018. In 2014, among 24829 organic farms, the most was found in the voivodeships: Warmia-Masuria, (4234), West Pomerania (3526) and Podlasie (3432). In total, the farms in these voivodeships constituted $45.1 \%$ of all organic farms in Poland. The lowest number of such farms was found in the voivodeships: Opole (75), Silesia (230) and Kuyavia-Pomerania (401), what constituted $2.8 \%$ in total. In 2018 , the most organic farms were found again in the voivodeships of Warmia-Masuria (3393) and Podlasie (2989), but the West Pomerania voivodeship (2060) was replaced by the Mazovia (2284). The share again amounted to $45.1 \%$. The lowest number of the organic farms again occurred in the voivodeships of Opole (61), Silesia (148) and Kuyavia-Pomerania (395), what constituted $3.1 \%$ of all organic farms in Poland. It is interesting that the situation in 2010 was the same in the group of voivodeships with the lowest number of the farms but diametrically different in the group of voivodeships with the highest number of the farms. In 2010, there were 79 organic farms in the Opole, 228 in the Silesian and 327 in the Kuyavia-Pomerania voivodeships what constituted $3.1 \%$. The set of the voivodeships with the highest share also contained, apart from the Warmia-Masuria (2279) and West Pomerania (2373), the mountain voivodeships: Subcarpathia (2091) and Lesser Poland (2156). In total, the organic farms in these units constituted $43.2 \%$ of all organic farms in Poland.

The most negative dynamics of changes in the share of the organic farms was observed in the Lesser Poland voivodeship - fall from $10.5 \%$ in 2010 to $4 \%$ in 2018 , as well as in Subcarpathia - fall from $10.2 \%$ in 2010 to $5.9 \%$ in 2018. The positive dynamics occurred in the Podlasie voivodeship - growth from $9.9 \%$ in 2010 to $15.6 \%$ in 2018, as well as in the Warmia-Masuria - growth from $11.1 \%$ in 2010 to $17.7 \%$ in 2018.

As the final conclusion, it must be emphasized that there are fewer and fewer farmers wanting to establish or transform their farms into organic ones. It is interesting in so far as the demand for organic products and healthy food is increasingly high. 


\section{Acknowledgements}

Paper published as a part of the project „VI Scientific and Technical Conference New Directions Of Research In Environmental Engineering, Energy, Geodesy And Spatial Economy" in Srebrna Gora, Poland.

\section{REFERENCES}

1. Biesiacki A., Kuś J., Madej A. 2004. Assessment of natural conditions for agricultural production, Wyd. IUNG Puławy

2. Dąbkowski N., Podawca K. 2017. Development of ecological farms in the Płock and Gostynin counties, Notatki Płockie : kwartalnik Towarzystwa Naukowego Płockiego, nr 4 (253), 18-24

3. Drabarczyk K., Wrzesińska-Kowal J. 2015. The development of organic farming in Poland, Zeszyty Naukowe Szkoły Głównej Gospodarstwa Wiejskiego, Ekonomika i Organizacja Gospodarki Żywnościowej nr 111, 19-31

4. Duda-Krynicka M., Jaskólecki H. 2010. The history and opportunities of organic farming in Poland, Problemy Ekologii, Vol. 14, nr 2, marzec-kwiecień 2010, str. 85-91

5. Golik D., Żmija D. 2017. Organic Farming and the Prospects for Its Development in Poland in the Light of the European Union's Experience, Zeszyty Naukowe Uniwersytet Ekonomiczny w Krakowie, 1 (961), s. 117-129

6. Kacprzak E., Kołodziejczak A. 2011. Development of organic farming in Poland over the years 2006-2009, Biuletyn IGSEiGP UAM, Seria Rozwój Regionalny i Polityka Regionalna, Nr 14. Poznań, 117-135

7. Komorowska D. 2007. Ecological farms in Poland in the light of data released by the Central Statistical Office GUS, Zeszyty Naukowe Szkoły Głównej
Gospodarstwa Wiejskiego w Warszawie, Problemy Rolnictwa Światowego, T. 2 (XVII), z. 2, 352-359

8. Kowalska A. 2010. The Determinants of Organic Agriculture Development in Poland and in Other European Countries, Annales Universitatis Mariae Curie-Skłodowska, sectio H, Oeconomia, 44 (XLIV), 47-63

9. Ligenzowska J. 2014. Organc farming in the world, Zeszyty Naukowe Szkoły Głównej Gospodarstwa Wiejskiego w Warszawie, Problemy Rolnictwa Światowego tom 14 (XXIX), zeszyt 3, 150-157

10. Łuczka-Bakuła W. 2013. The development of organic farming in Poland after its accession to the EU in the terms of the support from RDP 2004-2006, Rocz. Nauk. SERIA, t. XV, z. 1, 135-140

11. Makowska M., Gotkiewicz W., Pawlewicz A. 2015. Organic agriculture in spatial and environmental terms in Poland, Stowarzyszenie Ekonomistów Rolnictwa I Agrobiznesu, Roczniki Naukowe, tom XVII, Zeszyt 4, 160-165

12. Runowski H. 2009. Organic farming - progress or regress? Roczniki Nauk Rolniczych, Seria G, Tom 96, z. 4, 182-193

13. Stuczyński T., Jończyk K., Korzeniowska-Pucułek R., Kuś J., Terelak H. 2007. Natural conditions for organic agricultural production and its current state in Poland, Studia i Raporty IUNG-PIB, Puławy, z. 5, 55-78

14. The report on organic farming in Poland in 20152016, Agricultural and Food Quality Inspection, Główny Inspektorat Jakości Handlowej Artykułów Rolno-Spożywczych, Warszawa 2017

15. The report on organic farming in Poland in 2017 2018, Agricultural and Food Quality Inspection, Główny Inspektorat Jakości Handlowej Artykułów Rolno-Spożywczych, Warszawa 2019

16. Zegar J. 2009. From research on socially sustainable agriculture. Final report - synthesis and recommendations, Instytut Ekonomiki Rolnictwa i Gospodarki Żywnościowej, Państwowy Instytut Badawczy, nr 175, Warszawa, 1-104 